J Pain. 2015 June ; 16(6): 558-568. doi:10.1016/j.jpain.2015.03.003.

\title{
The interaction of patient race, provider bias, and clinical ambiguity on pain management decisions
}

\author{
Adam T. Hirsh ${ }^{a},{ }^{*}$, Nicole A. Hollingshead ${ }^{a}$, Leslie Ashburn-Nardo ${ }^{a}$, and Kurt Kroenke ${ }^{b, c, d}$ \\ aDepartment of Psychology, Indiana University - Purdue University Indianapolis, Indianapolis, IN, \\ USA \\ bVA HSR\&D Center of Excellence on Implementing Evidence-Based Practice, Roudebush VA \\ Medical Center, Indianapolis, IN, USA \\ CIndiana Regenstrief Institute, Inc., Indianapolis, IN, USA \\ ${ }^{\mathrm{d} D e p a r t m e n t}$ of Medicine, Indiana University School of Medicine, Indianapolis, IN, USA
}

\section{Abstract}

Although racial disparities in pain care are widely reported, much remains to be known about the role of provider and contextual factors. We used computer-simulated patients to examine the influence of patient race, provider racial bias, and clinical ambiguity on pain decisions. One hundred twenty nine medical residents/fellows made assessment (pain intensity) and treatment (opioid and non-opioid analgesics) decisions for 12 virtual patients with acute pain. Race (Black/ White) and clinical ambiguity (high/low) were manipulated across vignettes. Participants completed the Implicit Association Test and feeling thermometers, which assess implicit and explicit racial biases, respectively. Individual- and group-level analyses indicated that race and ambiguity had an interactive effect on providers' decisions, such that decisions varied as a function of ambiguity for White but not Black patients. Individual differences across providers were observed for the effect of race and ambiguity on decisions; however providers' implicit and explicit biases did not account for this variability. These data highlight the complexity of racial disparities and suggest that differences in care between White and Black patients are, in part, attributable to the nature (i.e., ambiguity) of the clinical scenario. The current study suggests that interventions to reduce disparities should differentially target patient, provider, and contextual factors.

\footnotetext{
(C) 2015 Published by The American Pain Society.

*Correspondence: Adam T. Hirsh, Ph.D., Department of Psychology, Indiana University - Purdue University Indianapolis, $402 \mathrm{~N}$. Blackford St., LD124, Indianapolis, IN 46202, USA. Phone: +1 (317) 274-6942. athirsh@iupui.edu.

Disclosures

The authors report no conflicts of interest. This work was supported by grants from the National Institutes of Health (R01MD008931) and the American Pain Society (Future Leaders in Pain Research) awarded to the first author. The content is solely the responsibility of the authors and does not necessarily represent the official views of the National Institutes of Health.

Publisher's Disclaimer: This is a PDF file of an unedited manuscript that has been accepted for publication. As a service to our customers we are providing this early version of the manuscript. The manuscript will undergo copyediting, typesetting, and review of the resulting proof before it is published in its final citable form. Please note that during the production process errors may be discovered which could affect the content, and all legal disclaimers that apply to the journal pertain.
} 


\section{Keywords}

Race; bias; disparities; decision-making

\section{Introduction}

Suboptimal pain care is common, especially for Black patients [43]. Several factors contribute to this disparity, including differences in pain sensitivity, patient preferences, healthcare access, and, potentially, provider biases [2,54]. Explicit biases are conscious and deliberate, whereas implicit biases are automatically activated with little conscious awareness $[16,25,26]$. Several theories of racial discrimination (e.g., $[18,20])$ propose that many individuals simultaneously hold divergent racial attitudes implicitly and explicitly; that is, they consciously disavow biases yet exhibit negative evaluations of Blacks on implicit measures. Contemporary discriminatory behavior is predicted more by individuals' implicit than explicit biases [48].

Two empirical studies found that implicit racial bias was not associated with racial disparities in pain assessment [28] or treatment [51]. However, these studies were small and, more importantly, may have reduced the effect of implicit bias [5,57] by using straightforward, unambiguous scenarios portrayed with a written vignette [51] or simple picture [28]. Conversely, situations that increase cognitive load - e.g., situations that are complex and/or ambiguous - elicit greater discrimination [5,6,52,54]. Indeed, ambiguity is a hallmark feature of pain care [7] that affects layperson and provider judgments, such that patients whose pain reports are inconsistent with objective findings are viewed suspiciously and considered to be in less need of treatment [55]. One of the few studies to examine cognitive load and health disparities found that under conditions of high load, providers were more likely to diagnose female patients with depression - a stereotypically female condition [45]. This retrospective study did not allow researchers to manipulate the variables of interest, assess provider stereotyping directly, or control for confounds. Stronger preliminary evidence is provided by Burgess et al [6] who found that male physicians were less likely to prescribe opioids to Black patients under high cognitive load but more likely to prescribe opioids to Black patients under low load; female physicians were more likely to prescribe opioids to Black patients regardless of cognitive load.

While studies have primarily focused on opioid treatment, other important aspects of care may also be susceptible to differential practices across race. Black pain patients may be more often referred for urine drug tests and to substance abuse specialists [29] and denied early prescription renewals [4]. Additionally, Black pain patients may be vulnerable to having briefer face-to-face interactions with their (primarily White) providers $[3,23,37,39,42,53]$. The implications of this time disparity are significant, as face-to-face time predicts patient outcomes, provider satisfaction, and reduced healthcare costs $[17,44,47]$.

The current study used Virtual Human (VH) technology and lens model methodology to examine the role of provider bias and contextual ambiguity in the care of White and Black pain patients. Our primary hypotheses were that (1) providers would be less likely to use 
opioid medications for Black vs. White patients, (2) this disparity would be more pronounced for providers higher vs. lower in implicit racial bias, and (3) the effect of patient race on provider opioid decisions would be greater under conditions of high vs. low clinical ambiguity. We also examined the relationship between the amount of time participants spent on each patient and their decisions across race and ambiguity conditions. Portions of this investigation were presented at the 2014 conference of the American Pain Society.

\section{Methods}

\section{Participants}

Participants were recruited from medical residency/fellowship programs across the United States via posted fliers (for local sites only), email, and word of mouth. Eligible participants were at least 18 years old, currently enrolled in an accredited medical residency/fellowship program in the United States, and involved in patient care at the time of the study. Medical residents/fellows were chosen because they provide patient care currently and will be fully independent physicians in the near future; thus, they provide meaningful and consequential data about patient, provider, and contextual factors that influence pain care. Also, as a practical matter, medical residents/fellows are often easier to recruit for research studies than are independent physicians. One hundred seventy one individuals contacted the investigators and expressed interest in the study. Of these, 21 did not provide any additional information that allowed us to determine their eligibility; thus, they did not complete the study. Six potential participants did not meet eligibility requirements ( 3 were not medical residents/ fellows, and 3 did not have access to an appropriate computer). Fifteen potential participants met eligibility requirements, were provided login credentials to access the website, but did not complete the study. This resulted in a final sample of 129 participants (75\% of the initial pool) who completed the study. Just over half of the participants were men (54\%), and the mean age was 29.6 years $(\mathrm{SD}=2.7)$. Approximately $56 \%$ self-identified as White, $26 \%$ as Asian, $7 \%$ as Hispanic, $2 \%$ as Black, and $9 \%$ as other. The most represented states of residence were Texas (35\%), Indiana (30\%), Michigan (12\%), and Illinois (10\%). Over 75\% of participants were currently providing care in an inpatient hospital or emergency room setting. Participants reported a wide range of clinical specialties; the most represented were anesthesiology (17\%), internal medicine (12\%), pediatrics (12\%), and psychiatry (12\%). Participants' reported average clinical experience with pain was 43.26 ( $\mathrm{SD}=21.88$; rated on a 0-100 VAS anchored at "not at all experienced" and "very experienced").

\section{Study Design and Procedure}

We used a lens model design and virtual human technology for this study. The lens model is an analogue method used to examine individual decision-making. Inherent to this model is the assumption that individuals make decisions by attending to and weighting available information (cues) [12]. Lens model studies typically present a series of profiles that contain cues that participants may use to make decisions. Each profile contains a unique combination of cues. In this study, we were interested in one patient cue (race: White vs. Black) and one contextual cue (clinical ambiguity: Low vs. High). Four unique patient profiles were needed to represent each possible cue combination once ( 2 levels of race $\times 2$ levels of ambiguity $=4$ ). To enhance the reliability of the decision-making data and 
maximize statistical power (see Statistical Analyses section below), we created 12 unique computer-simulated patients so that each cue combination was presented thrice.

Patient profiles consisted of a video and text vignette. We used computer-simulated patient videos created with FaceGen software. This virtual human software allowed us to develop high fidelity computer-simulated patients that display standardized empirically-validated facial expressions of pain. We manipulated the facial features associated with pain to create two prototype pain expressions - one representing high pain and one representing low pain $[13,49]$. We then "morphed" these expressions onto different computer-simulated patients, such that equivalent pain expressions were displayed by White and Black patients. This innovative feature confers a higher level of experimental control and realism than is possible with typical approaches (e.g., retrospective chart reviews, paper-pencil vignettes). We successfully used, standardized, and validated these patient stimuli in previous studies, and previous participants have rated the stimuli as highly realistic and reflective of actual clinical situations [33-36,58,59].

Each video was accompanied by a text vignette that contained information about the patient's presenting problem. Patients were described as presenting to the Emergency Department with acute pain due to either a long bone fracture or musculoskeletal low back injury. Patient-reported pain varied randomly across vignettes from 7 out of 10 pain to 9 out of 10 pain but always fell within the severe range of pain intensity [38]. Vital sign values were also included, but these varied minimally across patients and were always within normal limits. The specific text also varied across patients to increase study realism; e.g., different names were used, as well as specific features of the clinical situation. However, with the exception of race and clinical ambiguity, the information was equivalent across patients.

The patient profiles were presented in random order. Patient race was represented in the videos. Clinical ambiguity was categorized as high vs. low, and manipulated via pain etiology and congruence of facial expression and pain report. For patients in the high ambiguity condition, the vignette text described pain due to a musculoskeletal low back injury (e.g., acute onset of pain while lifting a heavy box). To further heighten the ambiguous nature of this condition, and because musculoskeletal low back pain often lacks objective evidence of pathology $[11,41]$, the text vignettes stated that the patients' physical exam findings were unremarkable. High ambiguity patients also presented with an incongruent facial expression and pain report; specifically, they displayed a "low pain" expression in the video but reported a high level of pain in the text (7-9 out of 10). For patients in the low ambiguity condition, the vignette text described pain due to a long bone fracture (e.g., radius fracture due to a fall) that was confirmed upon x-ray. Low ambiguity patients also presented with a congruent facial expression and pain report; specifically, they displayed a "high pain" expression in the video and reported a high level of pain in the text (7-9 out of 10). Patients' facial expressions were standardized so that all patients representing "high pain" and all patients representing "low pain" projected similar levels of pain. A sample text vignette from a high ambiguity patient is presented below, with parenthetical content representing text from a low ambiguity patient. 
Mr. Smith is 43 years old. He presents to the Emergency Department with sudden onset low back pain that occurred while lifting a heavy box earlier today [acute right wrist pain after falling in his yard]. He rates his pain as 9 out of $10 . \mathrm{He}$ describes it as sharp and throbbing, and reports it significantly interferes with his ability to walk and sit comfortably [is made worse when moving his arm and gripping objects with his hand]. He denies neurological symptoms such as bowel or bladder dysfunction [paresthesias such as numbness, tingling, or burning]. His physical exam is unremarkable except for moderate paralumbar tenderness and an antalgic gait [His X-ray report is positive for a left-sided distal radius fracture]. He states he has not taken anything for the pain. Mr. Smith denies significant past medical or surgical history and currently takes no medications. He has no absolute contraindications for common treatment options for acute pain.

Participants completed the study online by accessing a website using unique login credentials. First, participants provided informed consent and completed the demographics questionnaire. Next, they read instructions about how to complete the task and then viewed and made clinical decisions for the 12 unique patients. For each patient, participants made decisions about the patients' pain level and their likelihood of using 3 different analgesics (parenteral opioid, oral opioid, and oral non-opioid) to treat the patients' pain. Patient profiles were presented in random order. The study took approximately 1 hour to complete, and participants were compensated with a gift card. Study procedures were approved by our institutional review board.

\section{Measures}

Demographics Questionnaire-Participants provided information about their sex, age, and race/ethnicity, and state of residence. They also indicated their current practice setting and clinical specialty.

Treatment Decisions-For each of the 12 patients, participants were asked to: "Rate the level of pain that this patient is experiencing. ( 0 "no pain" - 100 "extreme pain")." For each patient, participants also responded to the following pain treatment item: "Rate the likelihood that you would use the following treatments to relieve the patient's $\underline{\text { current }}$ pain while in the Emergency Department." The treatment options consisted of (1) parenteral opioid analgesic (e.g., morphine, hydromorphone), (2) oral opioid analgesic (e.g., oxycodone, hydrocodone), and (3) oral non-opioid analgesic (e.g., acetaminophen, ibuprofen). Participants rated each treatment on separate 0-100 VASs anchored at "not at all likely" and "very likely."

Implicit Racial Bias-The Race Attitude Implicit Association Test (IAT [26]) asks participants to categorize facial images as Black vs. White and evaluative words (e.g., joy, horrible, pleasure, awful) as good vs. bad. On critical trials, participants press a designated key if the stimulus is a Black face or a good word and press another key if the stimulus is a White face or a bad word. On reverse trials, the categories Black and bad share a response key, and White and good share a key. The trial order is counterbalanced. The IAT score is equivalent to the difference in average response time on these 2 blocks of trials divided by the pooled standard deviation. This D algorithm is the most widely accepted method of 
calculating IAT scores given its superior measurement properties relative to the use of raw scores or other transformations [26]. Scores of .15, .35, and .65 are customary break points to indicate slight, moderate, and strong implicit preference for Whites over Blacks (https:// implicit.harvard.edu/implicit/demo/background/raceinfo.html). The underlying assumption is that concepts that are readily associated are sorted faster than concepts that are more weakly associated. Thus, faster responses to the White+Good/Black+Bad combined task compared to responses to the Black+Good/White + Bad combined task indicate a stronger association of White than of Black with good vs. bad. This response difference is interpreted as an implicit bias against Blacks. Despite controversy [19], the IAT demonstrates good reliability and validity [46]. A meta-analysis of its predictive validity found the IAT predicted prejudice and stereotyping more effectively than did self-report [27].

Explicit Racial Bias-Thermometer scales were used to assess participants' explicit racial bias. Participants indicated their feelings toward White and Black individuals on separate 0 (cold) - 10 (warm) scales. The relative difference in feelings toward Whites vs. Blacks is represented by the difference in ratings between the two groups (White-Black); higher scores indicate more negative evaluations of Blacks vs. Whites. Thermometer scales are a widely used, reliable, and precise way to assess feelings and attitudes toward different social groups $[1,40]$. The thermometers used in this study are consistent with those used in another study examining explicit and implicit racial biases [50].

\section{Statistical Analyses}

A principal concern of lens model studies is the ratio of profiles to cues. These designs must strike a balance between statistical power and participant burden. A 5:1 profile-to-cue ratio is needed to estimate stable regression coefficients and adequately power the individuallevel analyses [12]. In this study, we manipulated 2 cues of interest (patient race and clinical ambiguity) and created 12 unique patient profiles, resulting in a profile-to-cue ratio that exceeded the 5:1 recommendation. This ratio permitted each possible cue combination to be presented thrice, which further enhanced study power at both the individual (idiographic) and group (nomothetic) levels of analysis [12].

In accord with lens model methodology, we conducted both individual-level (idiographic) and group-level (nomothetic) analyses to determine the influence of patient race and clinical ambiguity on participants' pain assessment treatment decisions. Individual-level multiple regression analyses examined each individual participant's data. Patient race and clinical ambiguity were entered simultaneously as independent variables in step 1 of the model, and the race $\mathrm{X}$ ambiguity interaction term was entered in step 2. Participants' pain assessment and treatment decisions were the dependent variables in their respective models. The standardized regression coefficient (beta weight, $\beta$ ) for each independent variable indicates the unique contribution and relative importance of that variable in the participant's decision. To quantify the amount of variance accounted for by race and ambiguity in participants' decisions, the semi-partial correlation coefficients for each variable within each decision were squared, and descriptive statistics were used to summarize these values. 
Following the individual-level analyses, we conducted group-level analyses to examine the pain assessment and treatment decisions for the overall sample. Participants' decision ratings for each cue combination were combined, and average decision ratings were calculated within each variable for each of the 4 decision domains (e.g., for patient race, average pain assessment ratings were calculated separately for White and Black patients). We then used repeated measures analyses of variance (rANOVAs) with Bonferonnicorrected pairwise comparisons to examine the main effects of race and ambiguity and the race $\mathrm{X}$ ambiguity interaction.

To examine participants' implicit and explicit racial bias, we first used descriptive statistics to characterized participants' responses to the IAT and feeling thermometers. A one-sample $\mathrm{t}$-test was used to determine if the average IAT score significantly deviated from zero - a theoretically bias-free score. T-tests were used to examine differences in thermometer ratings for Whites and Blacks. Correlation analyses examined the relationship between implicit and explicit bias scores. Finally, to determine the extent to which racial bias played a role in participants' pain assessment and treatment decisions, we repeated the rANOVAs described above, with participants' IAT and thermometer scores included in the models.

Finally, we examined the amount of time participants' spent rating the patient vignettes. Using time-stamp data from the study website, we calculated the amount of time each participant viewed and rated each patient. We converted these time values to seconds and averaged them for each level of patient race (White vs. Black) and clinical ambiguity (low vs. high). We then used rANOVAs with Bonferonni-corrected pairwise comparisons to examine main and interaction effects for race, ambiguity, and bias (IAT and thermometer scores). Lastly, we used rANOVAs with Bonferonni-corrected pairwise comparisons to examine whether time spent (White vs. Black and low vs. high ambiguity) differed between participants who were and were not influenced by the manipulated cues (race and ambiguity).

Consistent with previous lens model studies examining pain decision-making [30,31,36], we examined the results of individual-level analyses at both $\mathrm{p}<.05$ and $\mathrm{p}<.10$. The significance level for all group-level analyses was set to $\mathrm{p}<.05$. Generalized eta-squared $\left(\eta_{G}^{2}\right)$ coefficients were calculated for effect size.

\section{Results}

The decision-making results are organized by assessment and treatment type. For each decision type, we conducted individual-level (idiographic) and group-level (nomothetic) analyses.

\section{Pain Assessment}

Race-Individual-level analyses indicated that six participants had a significant regression coefficient for patient race $(\mathrm{p}<.05$; range of $\beta:-.33-.51)$, and 6 others had a race coefficient that approached significance $(\mathrm{p}<.10)$, indicating that patient race reliably influenced their pain assessment ratings. More specifically, of these 12 participants, 8 gave higher pain ratings to Black patients and 4 gave higher ratings to White patients. Group- 
level analyses (Table 1) indicated that, on average, there was not a significant main effect of patient race on participants' pain assessment decisions.

Ambiguity-Ninety participants were significantly influenced by clinical ambiguity when rating patients' pain ( $\mathrm{p}<.05$; range of $\beta:-.99--.38)$, and another 7 had an ambiguity coefficient that approached significance $(\mathrm{p}<.10)$. All of these participants gave higher pain assessment ratings to patients in the low ambiguity condition (i.e., wrist fracture + congruent facial expression and pain report). Consistent with these individual-level results, group-level analyses indicated that low ambiguity patients were rated as being in significantly greater pain than high ambiguity patients $\left(\mathrm{p}<.001, \eta^{2}{ }_{\mathrm{G}}=.11\right)$.

Race X Ambiguity-For eleven participants ( 8 at $\mathrm{p}<.05$; range of $\beta:-.99-1.29$ ), race and ambiguity interacted to influence their pain ratings. Eight of these participants gave higher ratings to Blacks (vs. Whites) in the high ambiguity condition and higher ratings to Whites (vs. Blacks) in the low ambiguity condition. The other 3 participants showed the opposite pattern of results. Group-level analyses indicated a significant race $\mathrm{X}$ ambiguity interaction ( $\mathrm{p}<.001, \eta^{2}{ }_{\mathrm{G}}=.13$ ) such that in the high ambiguity situation, Black patients were perceived to be in more pain than Whites $(\mathrm{p}<.001)$, whereas in the low ambiguity situation, Whites were perceived to be in more pain than Blacks $(\mathrm{p}<.001)$. This interaction also indicated that the effect of ambiguity on participants' pain assessment rating was more pronounced for White than Black patients.

\section{Parenteral Opioid}

Race-Ten participants (4 at $\mathrm{p}<.05$; range of $\beta . .32-.69$ ) were reliably influenced by patient race when indicating their likelihood of giving a parenteral opioid in the ED. Nine participants were more likely to use this treatment for Black patients than for White patients. There was no significant main effect of patient race on parenteral opioid decisions at the group-level of analysis.

Ambiguity-Ninety-three participants ( 82 at $\mathrm{p}<.05$; range of $\beta:-1.00--.53$ ) gave different treatment ratings for high vs. low ambiguity patients. The vast majority $(\mathrm{n}=90)$ were more likely to give a parenteral opioid medication to patients presenting with a low ambiguity condition; this difference was also observed at the group-level of analysis $(\mathrm{p}<$. $\left.001, \eta_{\mathrm{G}}^{2}=.10\right)$.

Race X Ambiguity-Patient race and clinical ambiguity interacted to influence the treatment ratings for 15 participants ( 8 at $p<.05$; range of $\beta:-.90-.99$ ). Eight of these participants gave higher ratings to Blacks in the low ambiguity condition and higher ratings to Whites in the high ambiguity condition. The other 7 participants showed the opposite pattern of results. When the individual-level data were collapsed across the entire sample, a significant interaction emerged $\left(\mathrm{p}<.001, \eta^{2}{ }_{\mathrm{G}}=.11\right)$. Black patients received higher treatment ratings than White patients in the high ambiguity condition $(\mathrm{p}<.001)$; this race effect was reversed for the low ambiguity condition $(\mathrm{p}<.001)$. Considering this interaction from another perspective, ambiguity more strongly affected participants' parenteral opioid decisions for White than Black patients. 


\section{Oral Opioid}

Race-Thirteen participants ( 7 at $\mathrm{p}<.05$; range of $\beta:-.64-.53$ ) were reliably influenced by patient race when deciding to treat the patients' pain with an oral opioid in the ED. Eight of these participants were more likely to give an oral opioid to White patients than to Black patients. When examined at the group level, treatment ratings for Black and White patients did not significantly differ.

Ambiguity-Seventy-eight participants (67 at $\mathrm{p}<.05$; range of $\beta:-1.00-.99)$ made consistently different oral opioid decisions across the two ambiguity conditions. As with parenteral opioid decisions, most $(n=68)$ gave higher treatment likelihood ratings to low ambiguity patients than to high ambiguity patients. Moreover, low ambiguity patients received significantly higher ratings at the group level of analysis $\left(p<.001, \eta^{2}{ }_{G}=.05\right)$.

Race X Ambiguity-A significant race $\mathrm{X}$ ambiguity interaction was found for 17 participants ( 8 at $\mathrm{p}<.05$; range of $\beta:-1.34-1.13$ ). Similar to parenteral opioids, most $(\mathrm{n}=$ 13) of these participants gave higher oral opioid ratings to Blacks in the low ambiguity condition and higher ratings to Whites in the high ambiguity condition, whereas the remaining 4 participants gave higher ratings to high ambiguity Black patients and low ambiguity White patients. A significant race $\mathrm{X}$ ambiguity interaction also emerged when individual ratings were averaged across the sample $\left(\mathrm{p}<.001, \eta^{2} \mathrm{G}=.05\right)$. Black patients received higher treatment ratings than White patients in the high ambiguity condition $(\mathrm{p}<$. 001), whereas White patients received higher ratings in the low ambiguity condition ( $\mathrm{p}<$. 001). This interaction also indicated that the effect of ambiguity on oral opioid decisions was significant and robust for White but not Black patients.

\section{Oral Non-Opioid Analgesic}

Race-Twelve participants ( 5 at $\mathrm{p}<.05$; range of $\beta:-.66-.75$ ) made reliably different decisions for Black and White patients when rating their likelihood of using an oral nonopioid analgesic for the patients' pain. Ten of these participants gave higher treatment ratings to Black than to White patients. Despite these individual-level results, group-level analyses indicated no significant differences in participants' treatment decisions for Black and White patients.

Ambiguity-Thirty-nine participants ( 31 at $\mathrm{p}<.05$; range of $\beta:-.98-1.00$ ) were influenced by clinical ambiguity when making oral non-opioid treatment decisions. In contrast to the opioid treatment ratings, the majority $(n=34)$ gave higher non-opioid treatment ratings to patients in the high ambiguity condition than to patients in the low ambiguity condition. Similar results were obtained at the group-level, such that high ambiguity patients received significantly higher non-opioid treatment ratings than low ambiguity patients $\left(\mathrm{p}<.001, \eta_{\mathrm{G}}^{2}=.01\right)$.

Race X Ambiguity-For eleven participants ( 7 at $\mathrm{p}<.05$; range of $\beta:-1.35-1.23$ ), race and ambiguity interacted to influence their oral non-opioid treatment ratings. Six participants gave higher ratings to Blacks (vs. Whites) in the low ambiguity condition and higher ratings to Whites (vs. Blacks) in the high ambiguity condition. The other 5 
participants showed the opposite pattern of results. At the group-level, the nature of the significant race $\mathrm{X}$ ambiguity interaction for oral non-opioid analgesics $\left(\mathrm{p}<.001, \eta_{\mathrm{G}}^{2}=.01\right)$ differed from the opioid treatment ratings. Specifically, Black patients received higher nonopioid ratings than White patients in the low ambiguity situation $(\mathrm{p}<.001)$, whereas White patients received higher ratings in the high ambiguity situation $(\mathrm{p}<.001)$. Despite this different pattern of results, the interaction again indicated that the effect of ambiguity on participants' treatment ratings was significant and pronounced for White patients but not for Black patients.

\section{Significance of Race and Ambiguity Cues}

We quantified the amount of variance accounted for by patient race and clinical ambiguity cues by squaring their semi-partial correlation coefficients across the 4 decision domains (Table 2). Results of these calculations indicated that, on average, the amount of variance accounted for by patient race was $4 \%$ for pain assessment decisions, $4 \%$ for parenteral opioid decisions, $5 \%$ for oral opioid decisions, and $8 \%$ for oral non-opioid decisions. Clinical ambiguity had a stronger effect, accounting for an average of $52 \%$ of the variability in pain assessment decisions, $57 \%$ in parenteral opioid decisions, $44 \%$ in oral opioid decisions, and $24 \%$ in non-opioid decisions. The race $\mathrm{X}$ ambiguity interaction effect was similar in magnitude to the race main effect, accounting for an average of $4 \%$ of the variability in both pain assessment and parenteral opioid decisions, and 7\% of the variance in both oral opioid and non-opioid decisions. As indicated in Table 2 (see range values) there was considerable individual variability in the magnitude of these influences. For example, although patient race was of little influence for some participants when making parenteral opioid treatment decisions, it was highly influential for others and accounted for as much as $48 \%$ of the variability in their treatment ratings. Clinical ambiguity was more influential, on average, for participants' decisions regarding pain assessment and opioid (parenteral and oral) treatment than for decisions regarding non-opioid analgesia, whereas patient race was more influential for this later treatment domain than for the former 3 domains.

\section{Implicit and Explicit Bias}

The average IAT score (average difference in response time on the 2 blocks of trials) indicated a moderate-to-strong implicit preference for Whites over Blacks (mean $=.50$, sd $=.42$, which significantly deviated from a theoretically bias free score of zero $(\mathrm{t}(122)=$ $13.19, \mathrm{p}<.001)$. Similarly, responses to the feeling thermometers indicated that participants had significantly more positive feelings $(\mathrm{t}(128)=5.25, \mathrm{p}<.001, \mathrm{~d}=.46)$ toward Whites ( mean $=77.37, \mathrm{sd}=20.75)$ than Blacks $($ mean $=73.61, \mathrm{sd}=23.23)$. IAT and feeling thermometer (ratings for Whites minus Blacks) scores were positively correlated $(r=.24, p$ $<.01$ ), indicating that greater implicit preference for Whites (over Blacks) was associated with "warmer" feelings toward Whites (over Blacks). To examine the influence of participants' implicit and explicit biases on pain management decisions, we repeated the group-level rANOVAs presented above, with implicit and explicit bias scores included in the model. No significant main or interaction effects were observed for either IAT or thermometer items (all $\mathrm{p}$ values > .05), and the race and ambiguity results presented above did not appreciably change when bias scores were included. 


\section{Time Spent Making Decisions}

The results of rANOVAs indicated that participants spent significantly more time on White patient vignettes than on Black patient vignettes $(\mathrm{p}<.05)$, but similar amounts of time on low and high ambiguity patients $(\mathrm{p}>.05)$. This main effect should be considered in light of a significant race $X$ ambiguity interaction $\left(F(1,128)=14.58, p<.001, \eta^{2}{ }_{G}=.01\right)$. Pairwise comparisons indicated that in the high ambiguity situation, participants spent significantly more time rating White patients than Black patients, whereas in the low ambiguity situation, participants spent more time rating Black patients than White patients. Not only was the direction of the ambiguity effect different for White and Black patients, but the magnitude of this effect also differed. Specifically, ambiguity had a more pronounced effect on time spent rating Black patients than White patients. There were no significant main or interaction effects involving IAT or thermometer scores (all $\mathrm{p}$ values $>.05$ ).

To further interrogate these data, we compared participants who were significantly influenced by race $(n=59, p<.10)$ when making pain management decisions to those who were not influenced $(n=70, p>.10)$. Participants who were influenced by race spent significantly more time rating White than Black patients $(\mathrm{p}=.01)$, whereas a significant race difference in time spent was not found among participants who were not influenced by race $(\mathrm{p}>.05)$. Analyses of the ambiguity data indicated that participants who used ambiguity (n $=119, \mathrm{p}<.10)$ when making decisions spent significantly more time rating high vs. low ambiguity patients $(\mathrm{p}<.05)$, whereas participants who did not use ambiguity $(n=10, p>$. 10) evinced no significant time difference between high and low ambiguity patients $(\mathrm{p}>$. 05). These significant findings should be interpreted cautiously given the unbalanced nature of the groups.

\section{Discussion}

We used high-fidelity, virtual patient stimuli to examine the influence of patient race, clinical ambiguity, and provider racial bias on pain management decisions. A group-level significant interaction indicated that clinical ambiguity influenced providers' decisions for White but not Black patients. Individual differences among providers emerged for the effect of race and ambiguity on providers' decisions; however, provider racial bias did not account for this variability.

The results did not support our hypothesis that providers would be less likely to use opioids for Black patients. Previous clinical studies found White patients were more likely to receive opioids than Black patients [43]. Other studies, especially experimental ones, did not find racial disparities $[32,34,56]$. One potential explanation lies in methodology. Although previous clinical studies attempted to control for factors that may confound racial differences, accounting for all confounders is impossible. Despite gains in clinical relevance, this loss of experimental control reduces internal validity, thereby disallowing strong causal conclusions about racial differences. Our approach allowed us to manipulate race and ambiguity while holding other variables constant, thus maximizing internal validity even if not fully capturing the richness of actual clinical care. This is an important consideration for disparities research, particularly when attempting to draw conclusions across diverse studies with inconsistent results. Not all studies are created equal, nor should their results be equally 
weighted. One might reasonably privilege clinical studies that have high external validity. Nevertheless, experimental studies - especially those with enhanced realism - do inform our understanding of the causes of pain disparities.

This study is noteworthy for examining a race-by-ambiguity interaction. One interpretation of our findings is that Blacks received care that was less responsive to contextual information. Ambiguity characterizes many pain conditions and may or may not be relevant to treatment. We manipulated ambiguity via the presenting problem, objective findings, and facial expressions, all of which are relevant. Thus, it seems reasonable that providers were influenced by ambiguity, a pattern that was observed for White patients. However, for Black patients, providers' decisions were unchanged across ambiguity conditions, suggesting their care was less responsive to meaningful contextual information. Although this interpretation is consistent with findings that Blacks receive less optimal care than Whites [43], the raceby-ambiguity interaction could be interpreted differently. One could conclude that Black patients received more consistent care, whereas White patients received variable care and in some instances may have been over treated. Similar results were found by Chibnall et al $[9,10]$ where lumbar surgery rates for White but not Black Workers' Compensation claimants exceeded normative rates. Barring a clear standard of pain care, it is difficult to adjudicate these two interpretations. There is clear need for continued development of evidence-based clinical guidelines so that all patients may receive optimal care.

Additionally, future work should go beyond mere description of racial differences. For example, studies could further manipulate ambiguity to examine differences on this variable when the clinical information is less relevant to care than it was in this study. In accord with Aversive Racism Theory, one might hypothesize that treatment for Blacks would vary across irrelevant ambiguity manipulations - specifically, Blacks would receive less optimal care in high ambiguity situations, despite the fact that these ambiguity manipulations do not provide useful clinical information.

At first glance, the individual- and group-level interaction results conflict: although more participants gave higher opioid ratings to Blacks in the low ambiguity condition and Whites in the high ambiguity condition, the opposite pattern was significant at the group level. Two factors likely accounted for this apparent contradiction. The nature of the effect was split at the individual level ( 8 participants evinced the result described above, whereas 7 participants demonstrated the opposite pattern). This suggests the interaction effect, although statistically significant, was relatively smaller in magnitude for the 8 participants. The effect may have been more robust for the other 7 participants, so much so that it contributed to a significant group-level effect. It is also possible that non-significant individual-level effects summated to a significant group-level effect. Post-hoc examination of the data (not presented) suggested that both explanations contributed to the divergence of individual- and grouplevel results. Although not specific to race and ambiguity, similar "paradoxical" findings were recently reported [34]. Collectively, these findings highlight the importance of multilevel data analysis when investigating pain decision-making; sole reliance on group-level data may neglect important individual differences in treatment practices.

Another noteworthy feature of this study was its assessment of provider biases. Participants showed a moderate-to-strong implicit bias favoring Whites over Blacks and expressed more 
positive (explicit) feelings toward White than Black patients. These biases did not significantly predict pain decisions across patient race. Similarly, Sabin et al [51] and Haider et al [28] found that implicit racial bias was not associated with pain care provided by pediatricians and medical students, respectively. One might conclude that provider bias is not important. Another possibility concerns level of specificity. Like Sabin and Haider, we assessed general attitudes rather than specific stereotypic associations that might have a stronger bearing on care. For example, stereotypes that dehumanize Blacks might have a greater influence on pain decisions than the broad-based, general attitudes measured by feeling thermometers and the IAT. Just as the extent to which individuals implicitly associate Blacks with apes predicts their acceptance of violence toward Black suspects [21], including Black male children [22], so too might it predict the extent to which Blacks are perceived as capable of experiencing pain. In a study conducted after Hurricane Katrina, Whites perceived that Blacks were less likely than other Whites to experience uniquely human emotions such as anguish and remorse. In turn, the more such dehumanization occurred, the less Whites intended to provide aid to Black victims [14]. Together, these studies $[14,21,22]$ suggest that some providers may feel less moral imperative to provide quality care to Black than White patients. This speculation could be tested by examining these more specific racial stereotypes to understand their contribution to pain disparities. Studies should also examine other provider factors (e.g., demographic and clinical characteristics) that are hypothesized to influence pain care for diverse patients.

We also examined the amount of time participants spent rating patients, a potential indicator of "investment" in care. One might expect participants to spend more time rating White patients. Conversely, if participants were more suspicious of Black patients - e.g., concerned about drug diversion $[4,29]$ - one might expect them to spend more time rating Black patients. Our participants spent more time on Whites in the high ambiguity condition and more time on Blacks in the low ambiguity condition. Moreover, ambiguity had a more pronounced effect on time spent rating Black than White patients, which is counter to the results for opioid treatment decisions. Although our time spent results do not lend themselves to clear interpretation, they are consistent with the notion that conceptualizations of disparities in pain care should include both process (e.g., time) and outcome (e.g., treatment) variables. Future work is needed to understand these variables better and determine whether educational initiatives [e.g., 8,15] to improve provider decision-making and reduce disparities should target them individually or collectively.

Study limitations should be considered. First, because this study used VH patients and hypothetical scenarios, the results may not perfectly translate to pain care involving real patients. Relatedly, the study may have been face-valid and elicited socially desirable responding. Although many participants expressed awareness of the study purpose, their results did not appreciably differ from participants who expressed no awareness (data not presented). Second, race and ambiguity are only two factors that could influence pain management. Future studies should examine factors such as SES and patient preference that are likely relevant in this context. Third, we focused on ambiguity, per se, and did not attempt to independently isolate the effects of etiology and congruence. Studies that use a fully balanced design would provide a more nuanced understanding of ambiguity. Fourth, because we used an acute pain scenario, it is unclear to what extent these findings apply to 
chronic pain. Fifth, treatments other than opioid and non-opioid analgesics should be examined, as the processes examined herein may operate differently across treatments. Relatedly, the results concerning time spent raised more questions than answers; this study was intended to merely explore this process-oriented aspect of care so as to stimulate future work. Finally, our sample was composed of medical residents/fellows; thus, the results may not apply to other providers.

This study further elucidates the influence of patient, provider, and contextual factors on pain decisions. Given the prevalence and costs of pain, and the fact that providers see thousands of patients throughout their careers, there is a public health need for interventions that reduce disparities and improve pain care. Such interventions may benefit from the individual-level analyses employed herein, which allow for more precise targeting than onesize-fits-all approaches. These data also highlight the need to conceptualize pain disparities beyond differences in the prescription of opioid medications; attention should also be paid to the process of clinical care such as face-to-face time between patient and provider.

\section{Acknowledgments}

The authors thank Charnelle Free, Macey Miller, and Anna Squillace for assistance in data management.

\section{References}

1. Alwin DF. Feeling thermometers versus 7-point scales which are better? Socio Methods Res. 1997; 25:318-40.

2. Anderson KO, Green CR, Payne R. Racial and ethnic disparities in pain: causes and consequences of unequal care. J Pain. 2009; 10:1187-1204. [PubMed: 19944378]

3. Beach MC, Saha S, Korthuis PT, Sharp V, Cohn J, Wilson IB, Eggly S, Cooper LA, Roter D, Sankar A, Moore R. Patient-provider communication differs for black compared to white HIVinfected patients. AIDS and Behav. 2011; 15:805-811.

4. Becker WC, Starrels JL, Heo M, Li X, Weiner MG, Turner BJ. Racial differences in primary care opioid risk reduction strategies. The Annals of Family Med. 2011; 9:219-225.

5. Burgess DJ. Are providers more likely to contribute to disparities under high levels of cognitive load? How features of the health setting may lead to biases in decision making. Med Decis Mak. 2010; 30:246-57.

6. Burgess DJ, Phelan S, Workman M, Hagel E, Nelson DB, Fu SS, Widome R, van Ryn M. The effect of cognitive load and patient race on physicians' decisions to prescribe opioids for chronic low back pain: a randomized trial. Pain Med. 2014; 15:965-74. [PubMed: 24506332]

7. Burgess DJ, van Ryn M, Crowley-Matoka M, Malat J. Understanding provider contribution to race/ ethnicity disparities in pain treatment: insights from dual process models of stereotyping. Pain Med. 2006; 7:119-34. [PubMed: 16634725]

8. Burgess DJ, van Ryn M, Dovidio J, Saha S. Reducing racial bias among health care providers: lessons from social-cognitive psychology. J Gen Intern Med. 2007; 22:882-87. [PubMed: 17503111]

9. Chibnall JT, Tait RC, Andresen EM, Hadler NM. Clinical and social predictors of application for social security disability insurance by workers' compensation claimants with low back pain. J Occup Environ Med. 2006; 48:733-740. [PubMed: 16832231]

10. Chibnall JT, Tait RC, Andresen EM, Hadler NM. Race and socioeconomic differences in postsettlement outcomes for African American and Caucasian workers' compensation claimants with low back injuries. Pain. 2005; 114:462-472. [PubMed: 15777871] 
11. Chou R, Qaseem A, Owens DK, Shekelle P. Diagnostic imaging for low back pain: advice for high-value health care from the American college of physicians. Ann Intern Med. 2011; 154:1819. [PubMed: 21282698]

12. Cooksey, RW. Judgment analysis: theory, methods and applications. San Diego: Academic Press; 1996.

13. Craig, KD.; Prkachin, KM.; Grunau, RVE. The facial expression of pain. In: Turk, DC.; Melzack, R., editors. Handbook of pain assessment. New York: Guilford Press; 1992. p. 257-276.

14. Cuddy A, Rock M, Norton M. Aid in the aftermath of hurricane katrina: inferences of secondary emotions and intergroup helping. Group Processes \& Intergroup Relations. 2007; 10:107-118.

15. Devine PG, Forscher PS, Austin AJ, Cox WTL. Long-term reduction in implicit race bias: a prejudice habit-breaking intervention. J Exp Soc Psychol. 2012; 48:1267-1278. [PubMed: 23524616]

16. Dovidio JF, Kawakami K, Gaertner SL. Implicit and explicit prejudice and interracial interaction. J Personal and Soc Psychol. 2002; 82:62-8.

17. Dugdale DC, Epstein R, Pantilat SZ. Time and the patient-physician relationship. J Gene Intern Med. 1999; 14:34-40.

18. Evans JT. Dual-processing accounts of reasoning, judgment and social cognition. Ann Rev Psychol. 2008; 59:255-78. [PubMed: 18154502]

19. Fazio RH, Olson MA. Implicit measures in social cognition research: their meaning and use. Annual Rev Psychol. 2003; 54:297-327. [PubMed: 12172003]

20. Gaertner, SL.; Dovidio, JF. Prejudice, Discrimination, and Racism. Florida: Academic Press; 1986. The Aversive Form of Racism.

21. Goff PA, Eberhardt JL, Williams MJ, Jackson MC. Not yet human: implicit knowledge, historical dehumanization, and contemporary consequences. J Personal Soc Psychol. 2008; 94:292-306.

22. Goff PA, Jackson MC, Di Leone BA, Culotta CM, DiTomasso NA. The essence of innocence: consequences of dehumanizing Black children. J Personal Soc Psychol. 2014; 106:526-45.

23. Gordon HS, Street RL, Sharf BF, Kelly PA, Souchek J. Racial differences in trust and lung cancer patients' perceptions of physician communication. J Clin Oncol. 2006; 24:904-909. [PubMed: 16484700]

24. Greenwald AG, Banaji MR. Implicit social cognition: attitudes, self-esteem, and stereotypes. Psy Review. 1995; 102:4-27.

25. Greenwald AG, McGhee DE, Schwartz JKL. Measuring individual differences in implicit cognition: The Implicit Association Test. J Personal and Soc Psychol. 1998; 74:1464-80.

26. Greenwald AG, Nosek BA, Banaji MR. Understanding and using the Implicit Association Test: I. an improved scoring algorithm. J Personal Soc Psychol. 2003; 85:197-216.

27. Greenwald AG, Poehlman TA, Uhlmann E, Banaji MR. Understanding and using the Implicit Association Test: III. meta-analysis of predictive validity. J Personal Soc Psychol. 2009; 97:17-41.

28. Haider AH, Sexton J, Sriram N, Cooper LA, Efron DT, Swoboda S, Villegas CV, Haut ER, Bonds M, Pronovost PJ, Lipsett PA, Freischlag JA, Cornwell EE. Association of unconscious race and social class bias with vignette-based clinical assessments by medical students. JAMA. 2011; 306:942-51. [PubMed: 21900134]

29. Hausmann LRM, Gao S, Lee ES, Kwoh CK. Racial disparities in the monitoring of patients on chronic opioid therapy. Pain. 2013; 154:46-52. [PubMed: 23273103]

30. Hirsh AT, Alqudah AF, Stutts LA, Robinson ME. Virtual human technology: capturing sex, race, and age influences in individual pain decision policies. Pain. 2008; 140:231-238. [PubMed: 18930596]

31. Hirsh AT, Callander SB, Robinson ME. Patient demographic characteristics and facial expressions influence nurses' assessment of mood in the context of pain: a virtual human and lens model investigation. Int J Nurs Stud. 2011; 48:1330-8. [PubMed: 21596378]

32. Hirsh AT, George SZ, Robinson ME. Pain assessment and treatment disparities: a virtual human technology investigation. Pain. 2009; 143:106-113. [PubMed: 19269742] 
33. Hirsh AT, Hollingshead NA, Bair MJ, Matthias MS, Kroenke K. Preferences, experience, and attitudes in the management of chronic pain and depression: a comparison of physicians and medical students. Clin J Pain. 2014; 30:766-74. [PubMed: 24064934]

34. Hirsh AT, Hollingshead NA, Bair MJ, Matthias MS, Wu J, Kroenke K. The influence of patient sex, race, and depression on clinician pain treatment decisions. Euro J Pain. 2013; 17:1569-79.

35. Hirsh AT, Hollingshead NA, Matthias MS, Bair MJ, Kroenke K. The influence of patient sex, provider sex, and sexist attitudes on pain treatment decisions. J Pain. 2014; 15:551-59. [PubMed: 24576430]

36. Hollingshead NA, Matthias MS, Bair MJ, Hirsh AT. Impact of race and sex on pain management by medical trainees: a mixed-methods pilot study of decision-making and awareness of influence. Pain Med. 2014:1-11.

37. Jensen J, King A, Guntzviller L, Davis L. Patient-provider communication and low-income adults: age, race, literacy, and optimism predict communication satisfaction. Patient Education and Couns. 2010; 79:30-35.

38. Jensen MP, Chen C, Brugger AM. Interpretation of visual analog scale ratings and change scores: a reanalysis of two clinical trials of postoperative pain. J Pain. 2003; 4:407-14. [PubMed: 14622683]

39. Johnson R, Saha S, Arbelaez J, Beach M, Cooper L. Racial and ethnic differences in patient perceptions of bias and cultural competence in health care. J Gen Intern Med. 2004; 19:101-110. [PubMed: 15009789]

40. Kinder DR, Drake KW. Myrdal's prediction. Political Psychol. 2009; 30:539-68.

41. Koes BW, Van Tulder MW, Thomas S. Diagnosis and treatment of low back pain. Br Med J. 2006; 332:1430-4. [PubMed: 16777886]

42. Malat. Social distance and patients' rating of healthcare providers. J Health Soc Behav. 2001; 42:360-72. [PubMed: 11831137]

43. Meghani SH, Byun E, Gallagher RM. Time to take stock: A meta-analysis and systematic review of analgesic treatment disparities for pain in the United States. Pain Med. 2012; 13:150-74. [PubMed: 22239747]

44. Morrison I, Smith R. Hamster health care: time to stop running faster and redesign health care. $\mathrm{Br}$ Med J. 2000; 321:1541. [PubMed: 11124164]

45. Muroff JR, Jackson JS, Mowbray CT, Himle JA. The influence of gender, patient volume and time on clinical diagnostic decision making in psychiatric emergency services. Gen Hosp Psychiatry. 2007; 29:481-88. [PubMed: 18022040]

46. Nosek, BA.; Greenwald, AG.; Banaji, MR. The implicit association test at age 7: a methodological and conceptual review. In: Bargh, JA., editor. Social psychology and the unconscious: the automaticity of higher mental processes. New York: Psychology Press; 2007. p. 265-92.

47. Ogden J, Bavalia K, Bull M, Frankum S, Goldie C, Gosslau M, Jones A, Jumar S, Vasant K. "I want more time with my doctor": a quantitative study of time and the consultation. Family Pract. 2004; 21:479-483.

48. Pearson AR, Dovidio JF, Gaertner SL. The nature of contemporary prejudice: insights from aversive racism. Soc and Personal Psychol Compass. 2009; 3:314-38.

49. Prkachin KM. The consistency of facial expressions of pain: a comparison across modalities. Pain. 1992; 51:297-306. [PubMed: 1491857]

50. Rudman LA, Feinberg J, Fairchild K. Minority members' implicit attitudes: automatic ingroup bias as a function of group status. Soc Cog. 2002; 20:294-320.

51. Sabin JA, Rivara FP, Greenwald AG. Physician implicit attitudes and stereotypes about race and quality of medical care. Med Care. 2008; 46:678-85. [PubMed: 18580386]

52. Sherman JW, Macrae CN, Bodenhausen GV. Attention and stereotyping: cognitive constraints on the construction of meaningful social impressions. Euro Review Soc Psychol. 2000; 11:145-75.

53. Siminoff L, Graham G, Gordon N. Cancer communication patterns and the influence of patient characteristics: disparities in information-giving and affective behaviors. Patient Education and Couns. 2006; 62:355-360.

54. Tait RC, Chibnall JT. Racial/ethnic disparities in the assessment and treatment of pain: psychosocial perspectives. Am Psychol. 2014; 69:131-141. [PubMed: 24547799] 
55. Tait RC, Chibnall JT, Kalauokalani D. Provider judgments of patients in pain: seeking symptom certainty. Pain Med. 2009; 10:11-34. [PubMed: 18992039]

56. Tamayo-Sarver JH, Dawson NV, Hinze SW, Cydulka RK, Wigton RS, Albert JM, Ibrahim SA, Baker DW. The effect of race/ethnicity and desirable social characteristics on physicians' decisions to prescribe opioid analgesics. Acad Emerg Med. 2003; 10:1239-48. [PubMed: 14597500]

57. van Ryn M, Saha S. Exploring unconscious bias in disparities research and medical education. JAMA. 2011; 306:995-6. [PubMed: 21900142]

58. Wandner LD, Heft MW, Lok BC, Hirsh AT, George SZ, Horgas AL, Atchison JW, Torres CA, Robinson ME. The impact of patients' gender, race, and age on health care professionals' pain management decisions: an online survey using virtual human technology. Int J Nurs Studies. 2014; 51:726-33.

59. Wandner LD, Hirsh AT, Torres CA, Lok BC, Scipio CD, Heft MW, Robinson ME. Using virtual human technology to capture dentists' decision policies about pain. J Dental Res. 2013; 92:301-5. 


\section{Perspective}

This study examined the unique and collective influence of patient race, provider racial bias, and clinical ambiguity on providers' pain management decisions. These results could inform the development of interventions aimed at reducing disparities and improving pain care. 


\section{Highlights}

- We examined how patient race, provider bias, and clinical ambiguity influence pain care.

- Providers' decisions varied as a function of ambiguity for White but not Black patients.

- Provider racial bias was not associated with differences in treatment decisions. 


\section{Pain Assessment}

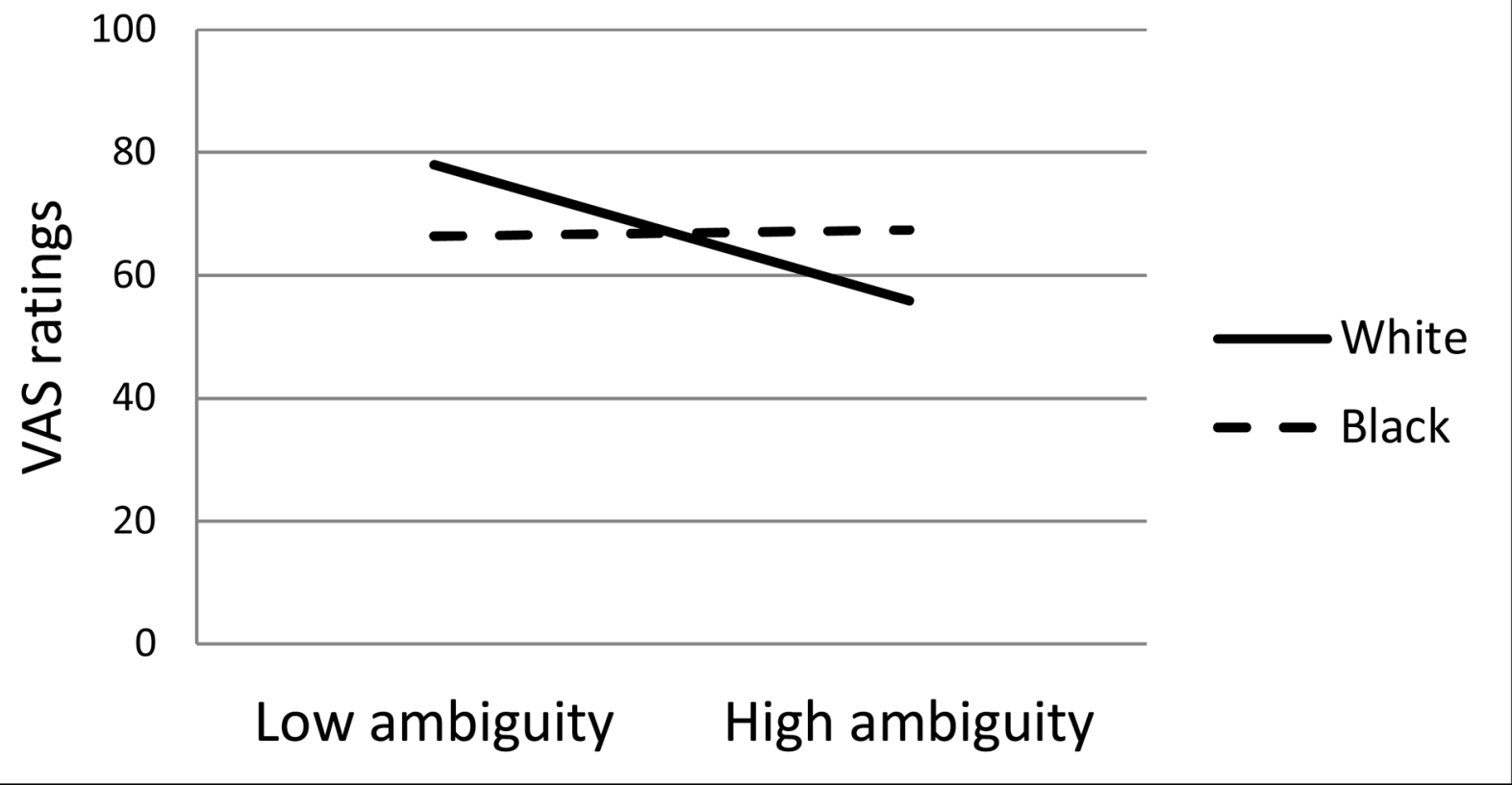

Figure 1.

Interaction of race and ambiguity on pain intensity ratings.

Note: VAS ratings made on 0 (no pain) - 100 (extreme pain) scale. 


\section{Parenteral Opioid}

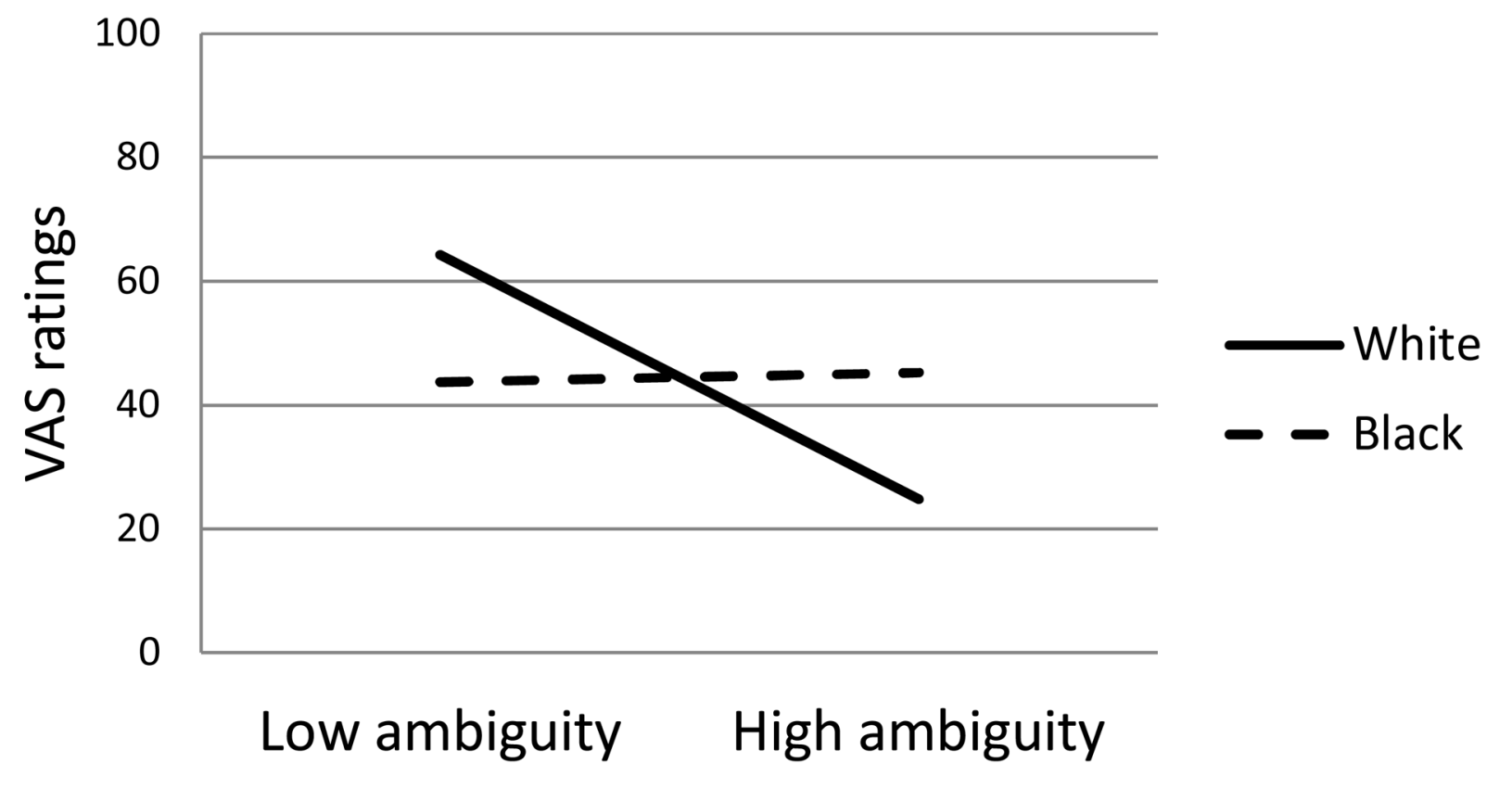

Figure 2.

Interaction of race and ambiguity on parenteral opioid ratings.

Note: VAS ratings made on 0 (not at all likely) - 100 (very likely) scale. 


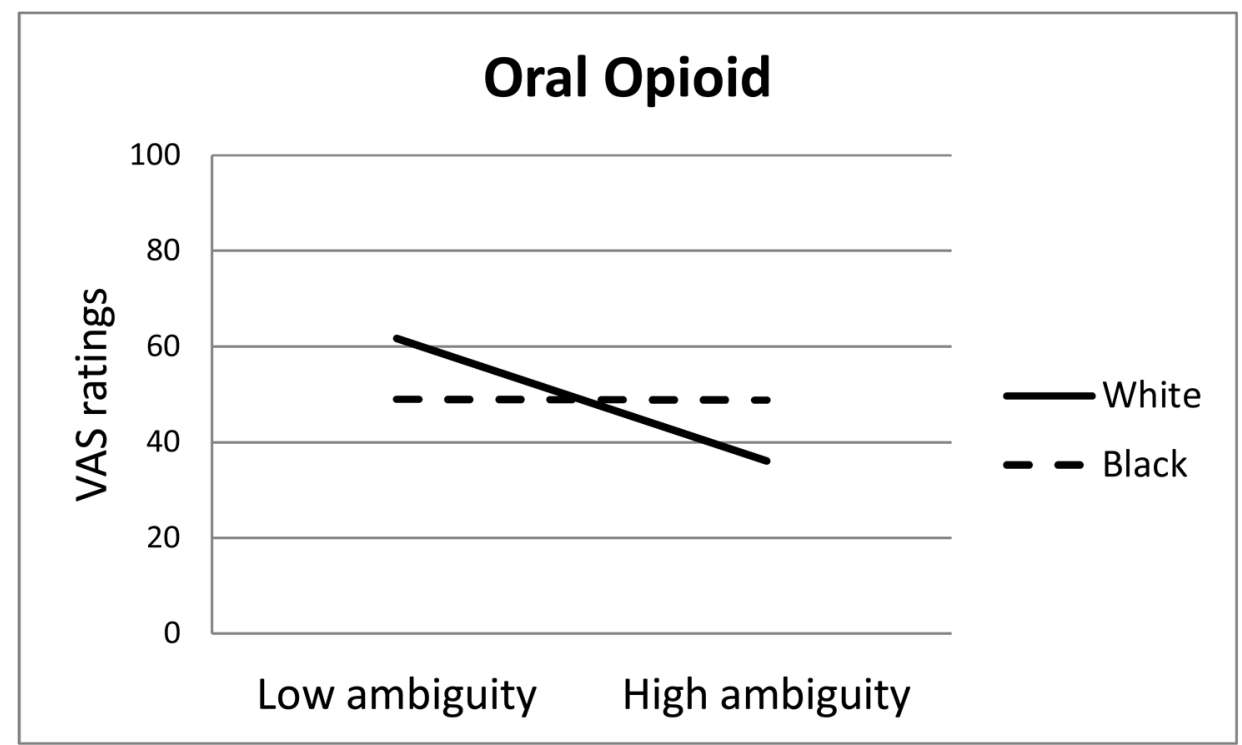

Figure 3.

Interaction of race and ambiguity on oral opioid ratings.

Note: VAS ratings made on 0 (not at all likely) - 100 (very likely) scale. 


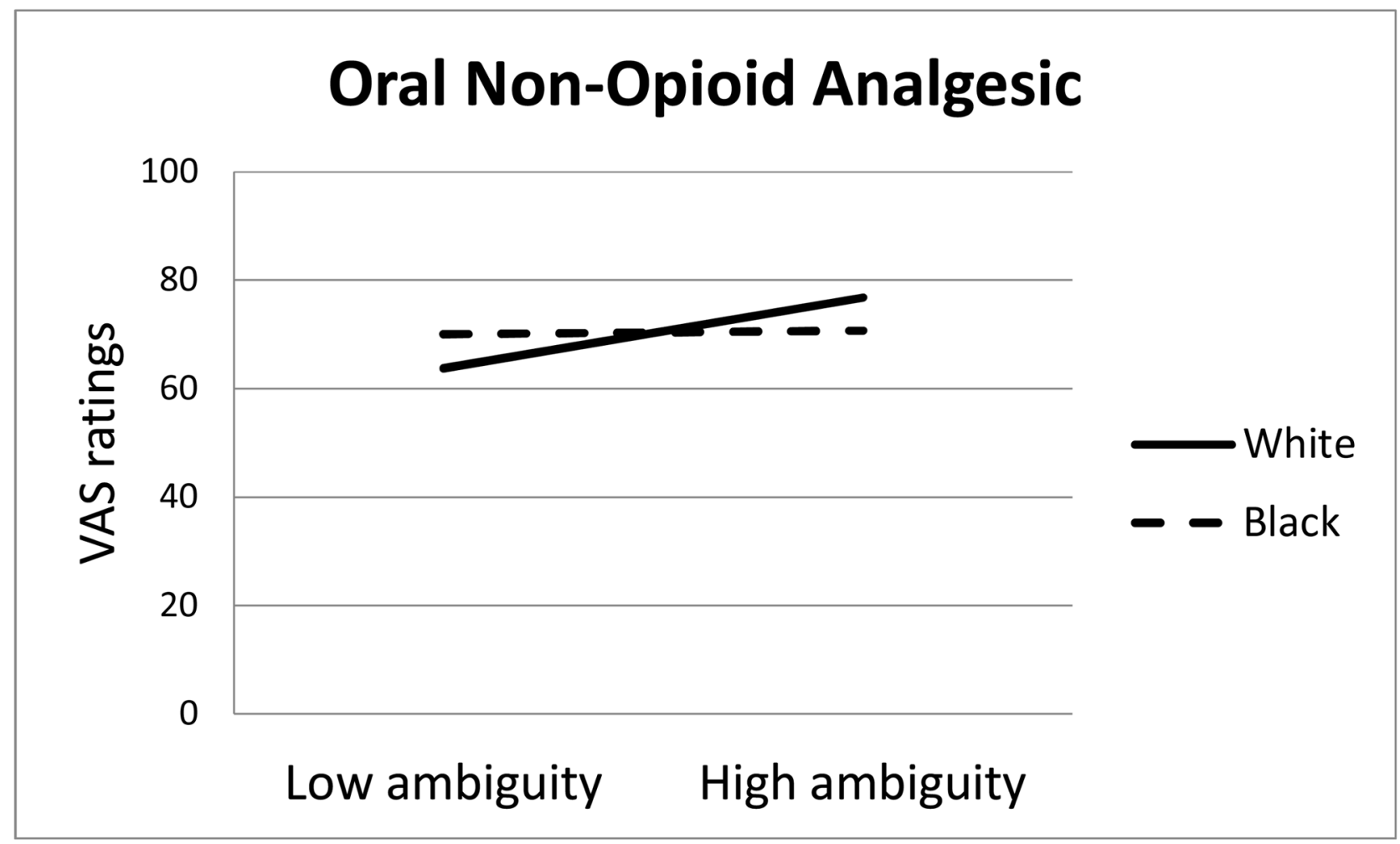

Figure 4.

Interaction of race and ambiguity on oral non-opioid ratings.

Note: VAS ratings made on 0 (not at all likely) - 100 (very likely) scale. 


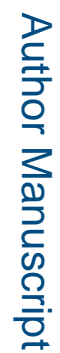

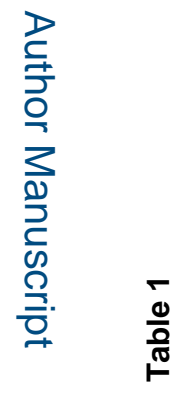

.0

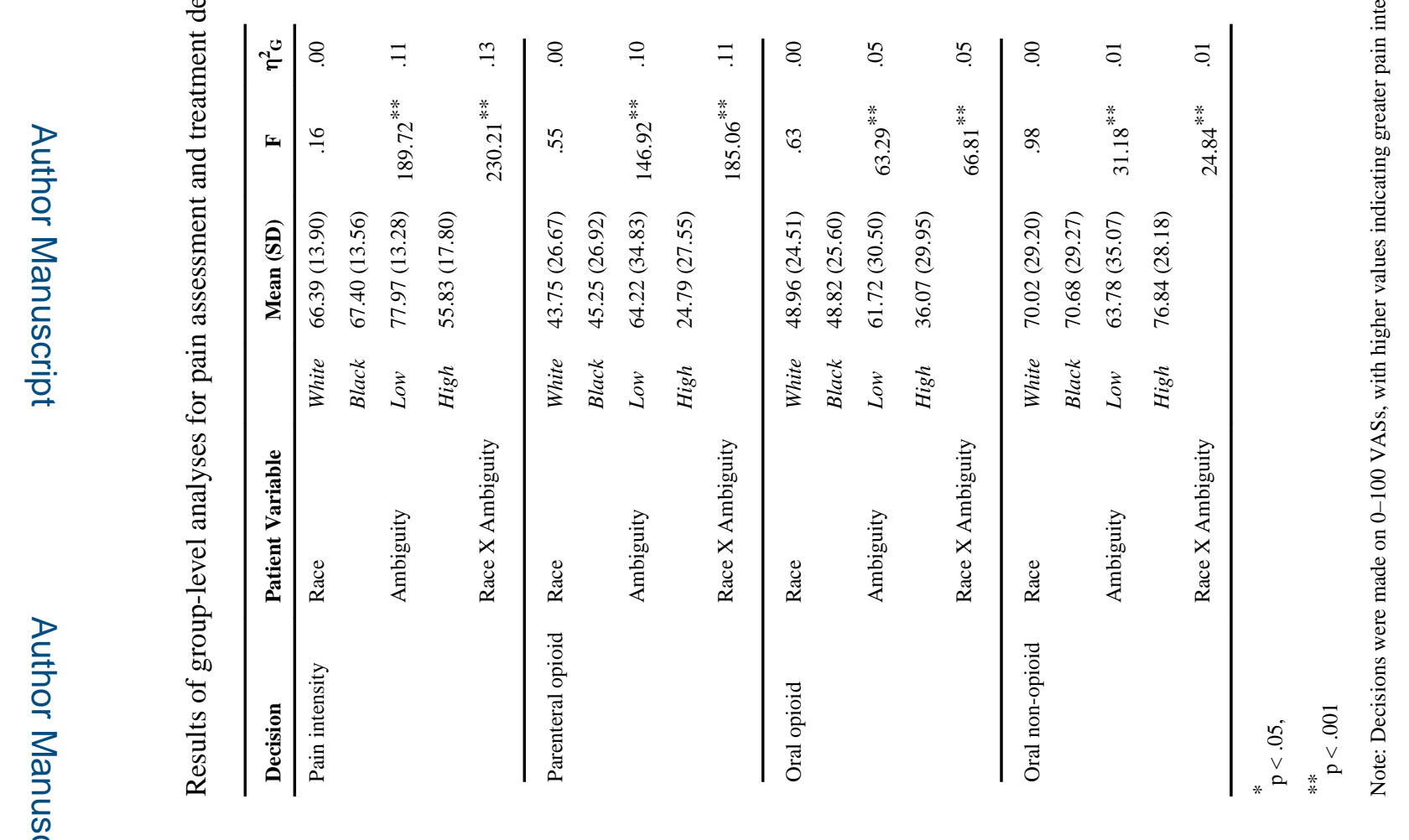

J Pain. Author manuscript; available in PMC 2016 June 01. 


\section{Table 2}

Variance in participants' decisions accounted for by race and ambiguity.

\begin{tabular}{llll}
\hline Treatment Decision & $\begin{array}{l}\text { Race } \\
\text { Mean (SD) } \\
\text { Range }\end{array}$ & $\begin{array}{l}\text { Ambiguity } \\
\text { Mean (SD) } \\
\text { Range }\end{array}$ & $\begin{array}{l}\text { Race X Ambiguity } \\
\text { Mean (SD) } \\
\text { Range }\end{array}$ \\
\hline Pain intensity & $0.04(0.06)$ & $0.52(0.31)$ & $.04(.08)$ \\
& $0.00-0.26$ & $0.00-0.99$ & $.00-.56$ \\
Parenteral opioid & $0.04(0.08)$ & $0.57(0.35)$ & $.04(.06)$ \\
& $0.00-0.48$ & $0.00-1.00$ & $.00-.33$ \\
Oral opioid & $0.05(0.07)$ & $0.44(0.36)$ & $.07(.10)$ \\
& $0.00-0.41$ & $0.00-1.00$ & $.00-.59$ \\
Oral non-opioid & $0.08(0.09)$ & $0.24(0.29)$ & $.07(.10)$ \\
& $0.00-0.56$ & $0.00-1.00$ & $.00-.61$ \\
\hline
\end{tabular}

Note: Values represent squared semi-partial correlation coefficients. 